Delft University of Technology

\title{
Active Resonator Reset in the Nonlinear Dispersive Regime of Circuit QED
}

Bultink, C. C.; Rol, M. A.; O'Brien, T. E.; Fu, X.; Dikken, B. C S; Dickel, C.; Vermeulen, R. F L; De Sterke, J. C.; Bruno, A.; Schouten, R. N.

DOI

10.1103/PhysRevApplied.6.034008

Publication date

2016

Document Version

Final published version

Published in

Physical Review Applied

\section{Citation (APA)}

Bultink, C. C., Rol, M. A., O'Brien, T. E., Fu, X., Dikken, B. C. S., Dickel, C., Vermeulen, R. F. L., De Sterke, J. C., Bruno, A., Schouten, R. N., \& DiCarlo, L. (2016). Active Resonator Reset in the Nonlinear Dispersive Regime of Circuit QED. Physical Review Applied, 6(3), 1-10. [034008].

https://doi.org/10.1103/PhysRevApplied.6.034008

Important note

To cite this publication, please use the final published version (if applicable).

Please check the document version above.

\section{Copyright}

Other than for strictly personal use, it is not permitted to download, forward or distribute the text or part of it, without the consent of the author(s) and/or copyright holder(s), unless the work is under an open content license such as Creative Commons.

\section{Takedown policy}

Please contact us and provide details if you believe this document breaches copyrights.

We will remove access to the work immediately and investigate your claim. 


\title{
Active Resonator Reset in the Nonlinear Dispersive Regime of Circuit QED
}

\author{
C. C. Bultink, ${ }^{1,2}$ M. A. Rol, ${ }^{1,2}$ T. E. O’Brien, ${ }^{3}$ X. Fu, ${ }^{1}$ B. C. S. Dikken,,${ }^{1,2}$ C. Dickel, ${ }^{1,2}$ R. F. L. Vermeulen,,${ }^{1,2}$ \\ J. C. de Sterke, ${ }^{4,1}$ A. Bruno, ${ }^{1,2}$ R. N. Schouten, ${ }^{1,2}$ and L. DiCarlo ${ }^{1,2}$ \\ ${ }^{1}$ QuTech, Delft University of Technology, P.O. Box 5046, 2600 GA Delft, The Netherlands \\ ${ }^{2}$ Kavli Institute of Nanoscience, Delft University of Technology, P.O. Box 5046, \\ 2600 GA Delft, The Netherlands \\ ${ }^{3}$ Instituut-Lorentz for Theoretical Physics, Leiden University, P.O. Box 9506, \\ NL-2300 RA Leiden, The Netherlands \\ ${ }^{4}$ Topic Embedded Systems B.V., P.O. Box 440, 5680 AK Best, The Netherlands \\ (Received 13 April 2016; revised manuscript received 10 July 2016; published 13 September 2016)
}

\begin{abstract}
We present two pulse schemes to actively deplete measurement photons from a readout resonator in the nonlinear dispersive regime of circuit QED. One method uses digital feedback conditioned on the measurement outcome, while the other is unconditional. In the absence of analytic forms and symmetries to exploit in this nonlinear regime, the depletion pulses are numerically optimized using the Powell method. We speed up photon depletion by more than six inverse resonator linewidths, saving approximately $1650 \mathrm{~ns}$ compared to depletion by waiting. We quantify the benefit by emulating an ancilla qubit performing repeated quantum-parity checks in a repetition code. Fast depletion increases the mean number of cycles to a spurious error detection event from order 1 to 75 at a $1-\mu$ s cycle time.
\end{abstract}

DOI: 10.1103/PhysRevApplied.6.034008

\section{INTRODUCTION}

Many protocols in quantum information processing require interleaving qubit gates and measurements in rapid succession. For example, current experimental implementations of quantum-error-correction (QEC) schemes [1-7] rely on repeated measurements of ancilla qubits to discretize and track errors in the data-carrying part of the system. Minimizing the QEC cycle time is essential to avoid a buildup of errors beyond the threshold for fault tolerance.

An attractive architecture for QEC codes is circuit quantum electrodynamics (cQED) [8]. Initially implemented with superconducting qubits, this scheme has since grown to include both semiconducting [9] and hybrid qubit platforms $[10,11]$. Readout in cQED involves dispersively coupling the qubit to a microwave-frequency resonator causing a qubit-state-dependent shift of the fundamental resonance. This shift can be measured by injecting the resonator with a microwave photon pulse. Inversely, however, resonator photons shift the qubit transition frequency (ac Stark shift [8]), leading to qubit dephasing and gate errors. To ensure photons leave the resonator before the gates recommence, QEC implementations include a waiting step after the measurement. During this dead time, lasting a significant part of the QEC cycle, qubits are susceptible to decoherence. While many prerequisites of measurement for QEC are already demonstrated (including a frequency-multiplexed readout via a common feed line [12], the use of parametric amplifiers to improve the speed and readout fidelity $[13,14]$, and null backaction on untargeted qubits [15]), comparatively little attention is given to the fast depletion of measurement photons.
Two compatible approaches to accelerate photon depletion have been explored. The first increases the resonator linewidth $\kappa$ while adding a Purcell filter $[2,16,17]$ to avoid enhanced qubit relaxation via the Purcell effect [18]. However, increasing $\kappa$ also enhances qubit dephasing (for a fixed ratio of the dispersive shift $\chi$ and $\kappa$ as desired for a high-fidelity readout $[19,20])$ by stray photons $[21,22]$, introducing a compromise. The second approach actively depletes photons using a counter pulse, as recently demonstrated by McClure et al. [23]. This demonstration uses symmetries available when the resonator response is linear. However, reaching the single-shot readout fidelity required for QEC often involves driving the resonator deep into the nonlinear regime, where no such symmetries are available.

In this paper, we propose and demonstrate two methods for active photon depletion in the nonlinear dispersive regime of $\mathrm{cQED}$. The first uses a homebuilt feedback controller to send one of two depletion pulses conditioned on the declared measurement outcome. The second applies a universal pulse independent of the measurement outcome. We maximize the readout fidelity at a measurement power 2 orders of magnitude larger than the power inducing the critical photon number in the resonator [8]. Missing analytic forms for this regime, we rely on numerical optimizations by Powell's method [24] to tune up pulses, defined by two or four parameters. Both depletion methods speed up depletion by at least approximately $1250 \mathrm{~ns}-5 / \kappa$ compared to waiting. To illustrate the benefits for QEC, we emulate an ancilla qubit performing parity checks [15,25] by subjecting our qubit to repeated rounds of coherent operations and measurement. We quantify the performance 
by extracting the mean number of rounds to an unexpected measurement outcome (i.e., a detection event). With active depletion, we observe an increase in this mean rounds to event, $\overline{\mathrm{RTE}}$, from 15 to 39 and reduce the cycle time to $1 \mu \mathrm{s}-4 / \kappa$. By further fixing the ancilla to remain in the ground state, the $\overline{\text { RTE }}$ increases to 75 . Simulations [26] indicate that, when including the same intrinsic coherence for surrounding data qubits, a five-qubit repetition code (studied in Ref. [2]) would have a logical error rate below its pseudothreshold [27].

\section{DEVICE CHARACTERIZATION}

We employ a 2D cQED chip containing ten transmon qubits with dedicated readout resonators, coupled to a common feed line (more details in Appendix A). We focus on one qubit-resonator pair for all data presented. This qubit has frequency $f_{q}=6.477 \mathrm{GHz}, T_{1}=25 \mu \mathrm{s}$, and $T_{2}^{\text {echo }}=39 \mu \mathrm{s}$. The resonator has a low-power fundamental at $f_{r,|0\rangle}=6.8506 \mathrm{GHz}\left(f_{r,|1\rangle}=6.8480 \mathrm{GHz}\right)$ for a qubit in $|0\rangle(|1\rangle)$, making the dispersive shift $\chi / \pi=-2.6 \mathrm{MHz}$. Note that this shift also corresponds to the qubit detuning per resonator photon. The fundamentals converge to the bare resonator frequency $f_{r \text {,bare }}=6.8478 \mathrm{GHz}$, at incident power $P_{\mathrm{rf}} \gtrsim-88 \mathrm{dBm}$. We calibrate a single-photon power $P_{\text {rf }}=-130 \mathrm{dBm}$ using photon-number-splitting experiments (Fig. 7) according to Ref. [28] and a critical photon number [8] $n_{\text {crit }}=\left(\Delta^{2} / 4 g^{2}\right) \approx 33\left(P_{\text {rf }} \approx-115 \mathrm{dBm}\right)$ using $f_{r,|0\rangle}-f_{r, \text { bare }}=g^{2} / 2 \pi \Delta$ and $\Delta=2 \pi\left(f_{q}-f_{r, \text { bare }}\right)$.

\section{MEASUREMENT TUNE-UP AND THE EFFECT OF LEFTOVER PHOTONS}

Our first objective is to maximize the average assignment fidelity of the single-shot readout:

$$
\mathcal{F}_{a}=1-\frac{1}{2}\left(\epsilon_{01}+\epsilon_{10}\right),
$$

where $\epsilon_{i j}$ is the probability of incorrectly assigning measurement result $j$ for input state $|i\rangle$. We map $\mathcal{F}_{a}$ as a function of the power $P_{\text {rf }}$ and frequency $f_{\text {rf }}$ of a measurement pulse of duration $\tau_{r}=1200 \mathrm{~ns}$ [Fig. 1(b)]. $\mathcal{F}_{a}$ is maximized at $P_{\mathrm{rf}}=-93 \mathrm{dBm}, 22 \mathrm{~dB}$ stronger than the $n_{\text {crit }}$ power. The nonlinearity is evidenced by the bending of resonator line shapes in the accompanying continuous-wave (cw) transmission spectroscopy [Fig. 1(a)]. We make two additions to further improve $\mathcal{F}_{a}$. First, we turn on a Josephson parametric amplifier (JPA), providing $14 \mathrm{~dB}$ of gain. The improved signal-to-noise ratio allows shortening $\tau_{r}$ to $300 \mathrm{~ns}$. Second, we use an optimized weight function (duration $\tau_{\text {int }}=400 \mathrm{~ns}$ ) to integrate the homodyne signal before thresholding. This weight function consists of the difference of the averaged transients for $|0\rangle$ and for $|1\rangle$ $[29,30]$. These additions achieve $\mathcal{F}_{a}=98.8 \%$, with $\epsilon_{01}=$ $0.1 \%$ and $\epsilon_{10}=2.3 \%$ [inset, Fig. 1(b)], limited by $T_{1}$.

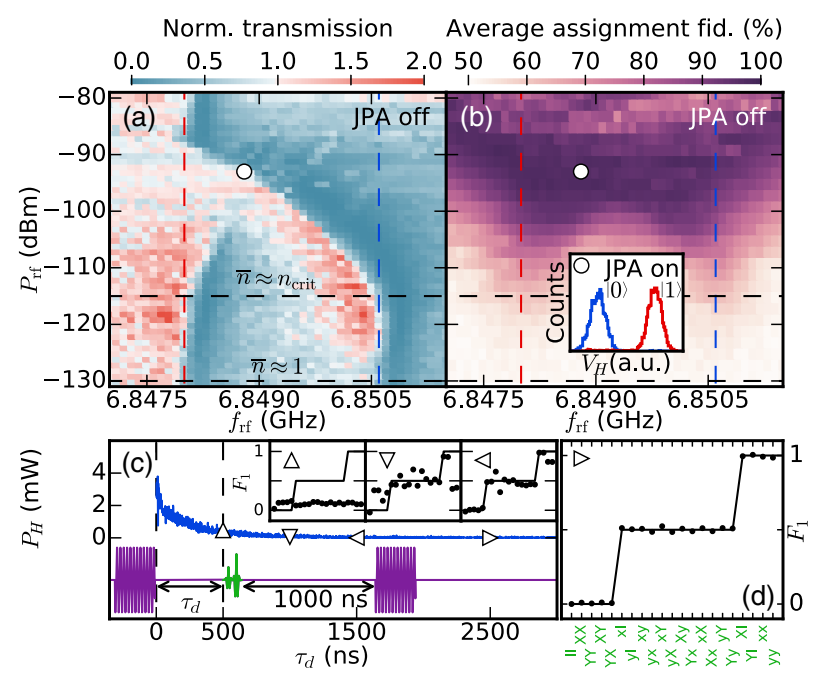

FIG. 1. (a) cw feed-line transmission spectroscopy as a function of incident power and frequency near the low- and high-power fundamentals of the resonator. The qubit is simultaneously driven with a weakly saturating tone. The right (left) vertical line indicates the fundamental $f_{r,|0\rangle}\left(f_{r,|1\rangle}\right)$ in the linear regime. The dot indicates $\left(P_{\mathrm{rf}}, f_{\mathrm{rf}}\right)=(-93 \mathrm{dBm}, 6.8488 \mathrm{GHz})$ used throughout the experiment. (b) Average assignment fidelity $\mathcal{F}_{a}$ as a function of $P_{\mathrm{rf}}$ and $f_{\mathrm{rf}}\left(\tau_{r}=1200 \mathrm{~ns}, \tau_{\mathrm{int}}=1500 \mathrm{~ns}\right)$, obtained from histograms with 4000 shots per qubit state. Inset: Turning on the JPA achieves $\mathcal{F}_{a}=98.8 \%$. (c) Illustration of qubit errors induced by leftover photons. At $\tau_{d}$, after an initial measurement pulse ends, AllXY qubit pulse pairs are applied and a final measurement is performed $1000 \mathrm{~ns}$ later to measure $F_{1}$. The transient of the decaying homodyne signal $P_{H}$ fits $1 / \kappa=250 \pm 2$ ns. Insets and $(\mathrm{d}): F_{1}$ versus the pulse pair for several $\tau_{d}$. The ideal two-step signature is observed at $\tau_{d} \gtrsim 2500 \mathrm{~ns}$.

The effect of this strong measurement on coherent operations is conveniently illustrated with AllXY measurements [31,32]. AllXY consists of 21 sequences, two pulses each [Fig. 1(d)], applied to the qubit followed by measurement. The pulses are drawn from the set $\{I, X, Y, x, y\}$, with $I$ the identity and $X$ and $Y(x$ and $y)$ denoting $\pi(\pi / 2)$ pulses around the $x$ and $y$ axis, respectively. Ideal pulses leave the qubit in $|0\rangle$ (first five pairs), on the equator of the Bloch sphere (next 12), and in $|1\rangle$ (final four), producing a characteristic two-step signature in the fidelity to $|1\rangle, F_{1}$ [Fig. 1(d)]. Distinct signatures reveal errors in many gate parameters [32]. Here, we apply an extra measurement pulse ending at time $\tau_{d}$ before the AllXY pulse pair to reveal the effect of leftover photons [Fig. 1(c)]. At $\tau_{d} \sim 7 / \kappa$, the characteristic signature of moderate qubit detuning is observed. At $\tau_{d} \leq 2 / \kappa$, the detuning is significant with respect to the Rabi frequency of pulses, which thus barely excite the qubit.

\section{AllXY AS A PHOTON DETECTOR}

To find depletion pulses, we rely exclusively on optimization with Powell's method and calibrate AllXY as our 
photon detector. We choose $\mathcal{E}_{\text {AllXY }}$ as the cost function, defined as the sum of the absolute deviations from the ideal two-step result. We find experimentally that $\mathcal{E}_{\text {AllXY }}=$ $\alpha \bar{n}\left(\tau_{d}\right)+\beta$ for average photon numbers $\bar{n} \lesssim 30$. The calibration of coefficients $\alpha$ and $\beta$ is described in Appendix B. Measurement noise limits the detector to $\delta \bar{n} \gtrsim 0.3$, providing a dynamic range of 2 orders of magnitude, suitable for the optimizations that follow.

\section{TUNE-UP AND COMPARISON OF TWO METHODS FOR ACTIVE PHOTON DEPLETION}

Our first depletion method uses a feedback controller to apply one of two depletion pulses, $D_{j}$, conditioned on the declared measurement result $j \in\{0,1\}$ [Fig. 2(a)]. The pulse $D_{j}$, a square pulse of duration $\tau_{p}=30 \mathrm{~ns}$, is applied at $f_{r,|j\rangle}$ by sideband modulating $f_{\mathrm{rf}}$. The combined delays from round-trip signal propagation ( $80 \mathrm{~ns}$ ), the augmented integration window (100 ns), and controller latency

$\begin{array}{ll}\text { (a) Conditional depletion } & \text { (c) Unconditional depletion }\end{array}$

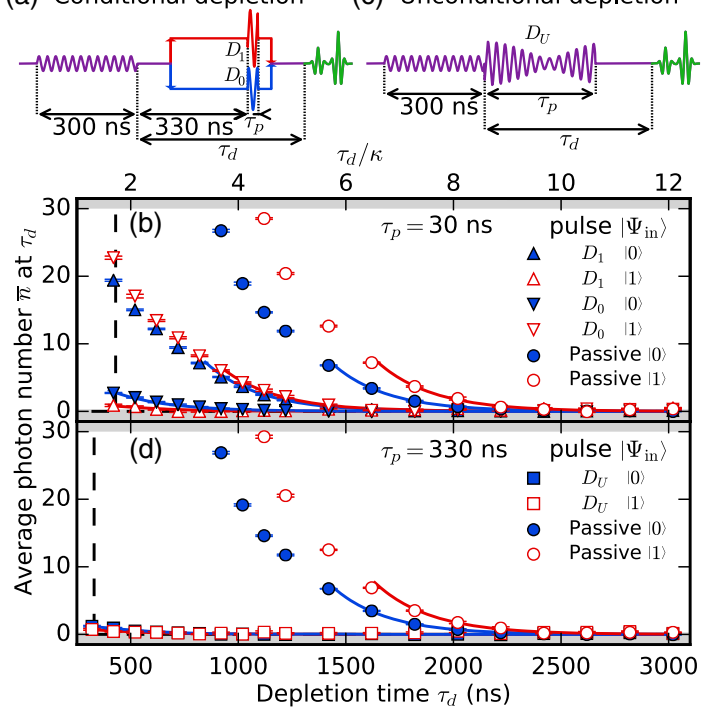

FIG. 2. (a) Pulse scheme for the conditional photon depletion. The controller applies a depletion pulse $D_{0}\left(\right.$ at $\left.f_{r,|0\rangle}\right)$ or $D_{1}$ (at $f_{r,|1\rangle}$ ), each with a separate amplitude and phase, depending on its declared measurement outcome. (b) Performance of the conditional depletion. Average photon number $\bar{n}$ as a function of $\tau_{d}$ for all combinations of input qubit state and depletion pulse. Compared to waiting, conditional depletion saves approximately 1250(1800) ns for correct declaration 0 (1). (c) Pulse scheme for the unconditional active depletion. The single depletion pulse $D_{U}$, immediately following the nominal measurement pulse, has four parameters corresponding to the amplitude and phase of two pulse components at $f_{r,|0\rangle}$ and $f_{r,|1\rangle}$. The summation of the two square pulse components produces the displayed beating at frequency $\left(f_{r,|0\rangle}-f_{r,|1\rangle}\right) / 2=\chi / 2 \pi$. (d) Performance of the unconditional depletion. Unconditional depletion saves approximately $1650(1900) \mathrm{ns}$ for $|0\rangle(|1\rangle)$. Exponential best fits (curves) to the data in the linear regime $(\bar{n} \leq 8)$ give $1 / \kappa=255 \pm 5 \mathrm{~ns}$.
(150 ns) make $D_{j}$ arrive $330 \mathrm{~ns}$ after the measurement pulse ends. Each pulse is separately optimized with the amplitude and phase as free parameters using a two-step procedure. We first minimize $\bar{n}$ at $\tau_{d}=1000 \mathrm{~ns}$ with the qubit initialized in $|i\rangle$. This $\tau_{d}$ is sufficiently long to avoid saturating the detector, and the sensitivity limit is reached after a few optimization rounds (further details on the optimization in Appendix C). A second optimization at $\tau_{d}=500 \mathrm{~ns}$ further optimizes the resulting pulse and converges to $\bar{n} \sim 2.1(0.7)$ for $|0\rangle(|1\rangle)$, reducing $\tau_{d}$ by at least $5 / \kappa$ compared to passive depletion [Fig. 2(b)]. An incorrect assignment by the feedback controller leads to less-effective depletion but still outperforms passive depletion.

Our second depletion method is unconditional (as in Ref. [23]), using a universal depletion pulse $D_{U}$ starting immediately after the measurement pulse [Fig. 2(c)]. To cope with the asymmetry of the nonlinear regime, we compose $D_{U}$ by summing two square pulses of duration $\tau_{p}=330 \mathrm{~ns}$ with independent amplitude and phase at $f_{r,|0\rangle}$ and $f_{r,|1\rangle}$. These four parameters are found minimizing the sum of $\bar{n}$ for $|0\rangle$ and $|1\rangle$, using a similar two-step procedure as for the conditional pulses (using $\tau_{d}=400 \mathrm{~ns}$ in the second step). This achieves $\bar{n} \sim 0.8(0.4)$ for $|0\rangle(|1\rangle)$ and reduces $\tau_{d}$ by $>6 / \kappa$ compared to passive depletion [Fig. 2(d)]. We do not currently understand why unconditional depletion outperforms conditional depletion and why depletion for $|1\rangle$ outperforms depletion for $|0\rangle$. Numerical studies of the depletion performance currently pursued outside our group [33] may soon help explain these observations and suggest other pulse parameterizations to achieve better depletion.

\section{BENCHMARKING DEPLETION METHODS WITH A QEC EMULATION: A FLIPPING ANCILLA}

We quantify the merits of these active depletion schemes with an experiment motivated by current efforts in QEC. Specifically, we emulate an ancilla qubit undergoing the rapid succession of interleaved coherent interaction and measurement steps when performing repetitive parity checks on data qubits in a repetition code [Fig. 3(a)]. We replace each conditional-phase gate with idling for an equivalent time (40 ns), reducing the coherent step to a 200-ns echo sequence that ideally flips the ancilla each round. As a performance metric, we measure the average number of rounds to an event, $\overline{\mathrm{RTE}}$. An event is marked by the first qubit measurement outcome deviating from the expected. Imperfections reducing the $\overline{\mathrm{RTE}}$ include qubit relaxation, dephasing and detuning during the interaction step, and measurement errors due to readout discrimination infidelity $1-\mathcal{F}_{d}$ (defined as the overlap fraction of Gaussian best fits to the single-shot readout histograms [17]). 
(a)

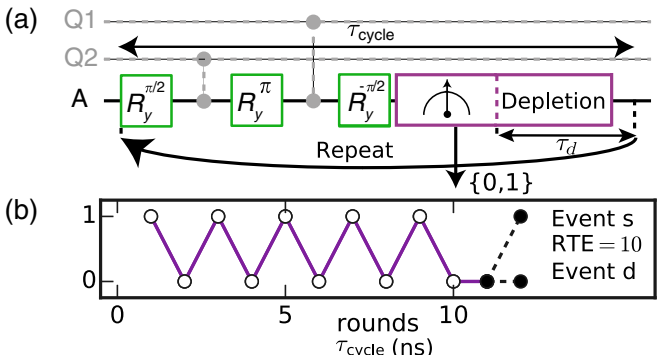

(c) $\quad \begin{array}{llllll}1000 & 1500 & 2000 & 2500 & 3000 & 3500\end{array}$

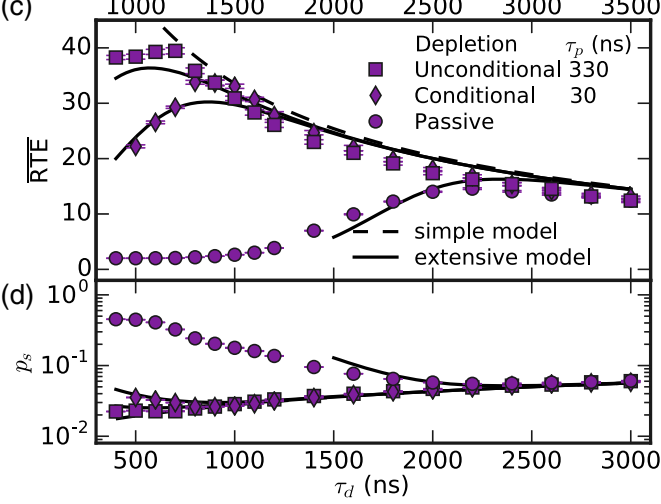

FIG. 3. (a) Block diagram for parity measurements in a repetition code. The ancilla $A$ performs an indirect measurement of the parity of data qubits $Q_{1}$ and $Q_{2}$ by a coherent interaction step followed by measurement. This emulation replaces conditional-phase gates by idling, reducing the coherent step to an echo sequence that ideally flips the ancilla. The measurement step is followed by a depletion step of duration $\tau_{d}$, after which a new cycle begins. (b) Single trace of digitized measurement outcomes. The counting of rounds is ended by two types of event, $s$ and $d$. (c) Average rounds to an event as a function of $\tau_{d}$. The unconditional method improves the $\overline{\mathrm{RTE}}$ by a factor of 2.7 and reduces the optimum $\tau_{d}$ by a factor of 2.7. (d) Per-round probability of a type-s event versus $\tau_{d}$. Added curves are obtained from the two models described in Appendix E.

To differentiate these sources of ancilla hardware errors, we distinguish two types of detection events, determined by the measurement outcome in the round following the first deviation [Fig. 3(b), similar to Ref. [34]]. Events of type $s$ can result, for example, from one ancilla bit flip or from measurement errors in two consecutive rounds. In turn, events of type $d$ can result from one measurement error or from ancilla bit flips in two consecutive rounds. Because photon-induced errors primarily lead to single bit flips, we also extract the probability of encountering an event of type $s$ per cycle, $p_{s}$, and investigate its $\tau_{d}$ dependence.

Decreasing $\tau_{d}$ trades off $T_{1}$-induced errors for photoninduced errors. For passive depletion, the $\overline{\mathrm{RTE}}$ is maximized to 14.6 at $\tau_{d}=2200 \mathrm{~ns}$ [Fig. 3(c)]. At this optimum, depletion occupies most of the total QEC cycle time $\tau_{\text {cycle }}=2700 \mathrm{~ns}$. Both active depletion methods reach a higher $\overline{\mathrm{RTE}}$ by balancing the trade-off at lower $\tau_{d}$. As in the

optimization, we find that the unconditional depletion performs best, improving the maximal $\overline{\mathrm{RTE}}$ to 39.5 at $\tau_{d}=700 \mathrm{~ns}$ when $\bar{n} \sim 0.29(0.14)$ for $|0\rangle(|1\rangle)$, which reduces the optimum $\tau_{\text {cycle }}$ to $1200 \mathrm{~ns}$.

The essential features of the $\overline{\mathrm{RTE}}$ for the three depletion schemes are well captured by two theory models (detailed description in Appendix E). The simple model includes only qubit relaxation and non-photon-induced dephasing (calibrated using standard $T_{1}$ and $T_{2}^{\text {echo }}$ measurements). The extensive model also includes photoninduced qubit dephasing and detuning during the coherent step (modeled following Ref. [35] with the photon dynamics of Fig. 2) and a measured $1-\mathcal{F}_{d}=0.1 \%$ for readout. As we do not model qubit gate errors, we restrict the extensive model to $\bar{n}<8$. The good agreement between the extensive model and experiment confirms the $\bar{n}$ calibration and demonstrates the nondemolition character of the measurement. The conditions for a nondemolition readout in the nonlinear regime have recently been investigated in Ref. [36].

\section{OPTIMIZATION OF THE DEPLETION PULSE LENGTH}

In attempts to further shorten the depletion time, we explore depletion for various pulse lengths, finding smooth variation in optimal pulse parameters but no significant improvement of the $\overline{\mathrm{RTE}}$ (Fig. 4). For a variety of $\tau_{p}$, the optimized pulse amplitudes and phase parameters are shown, along with the residual photon number and results for multiround QEC emulation. For the conditional depletion, the optimal amplitude $A_{0}\left(A_{1}\right)$ of $D_{0}\left(D_{1}\right)$ decreases smoothly as $\tau_{p}$ increases, whereas the optimal phase $\phi_{0}\left(\phi_{1}\right)$ remains constant. The residual $\bar{n}$ and readout discrimination infidelity do not show any dependence on $\tau_{p}$. As expected, there is no dependence of $\mathcal{F}_{d}$ on $\tau_{p}$, as there is no overlap between the depletion pulse and integration window. The $\overline{\mathrm{RTE}}$ and per-round probability of a type-s event for emulated QEC in the flipping configuration do not show any dependence on $\tau_{p}$ either. For the unconditional depletion, the optimal values of the four parameters, defining the universal depletion pulse $D_{U}$, evolve smoothly as $\tau_{p}$ is varied. The residual $\bar{n}$ first decreases weakly with decreasing $\tau_{p}$ but increases sharply for $\tau_{p}<250 \mathrm{~ns}$. A smooth decrease in $\mathcal{F}_{d}$ is observed for decreasing $\tau_{p}$. We attribute this effect to the overlap between $D_{U}$ and the measurement integration window. We note that a slightly higher $\overline{\text { RTE }}$ might be achieved by implementing a short wait time between the measurement pulse and the depletion pulse to combine the lower achieved $\bar{n}$ for $\tau_{p}=270$ to $315 \mathrm{~ns}$ with the higher $\mathcal{F}_{d}$ of the longer pulses. However, we do not explore this experimentally. 


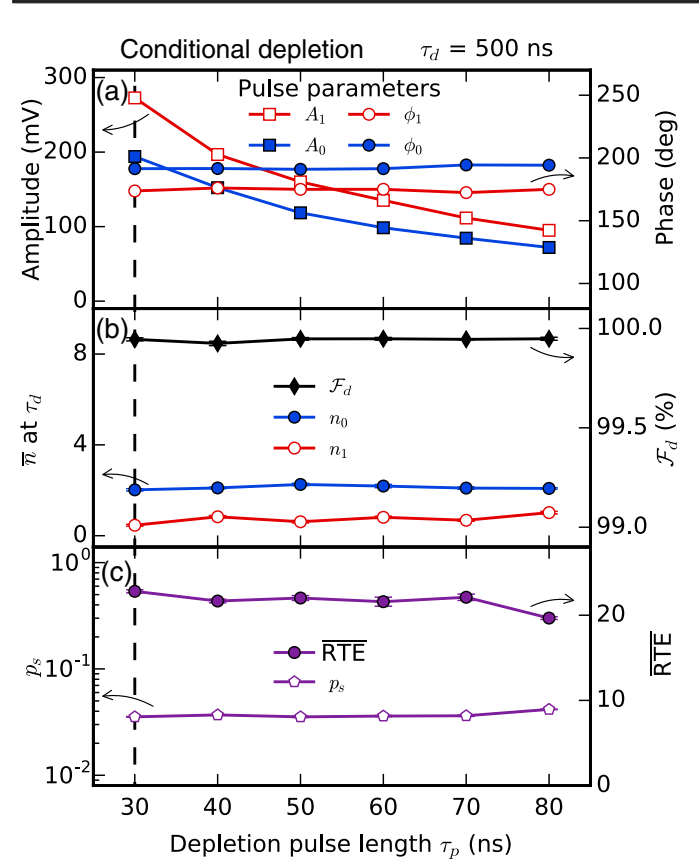

Depletion pulse length $\tau_{p}$ (ns)

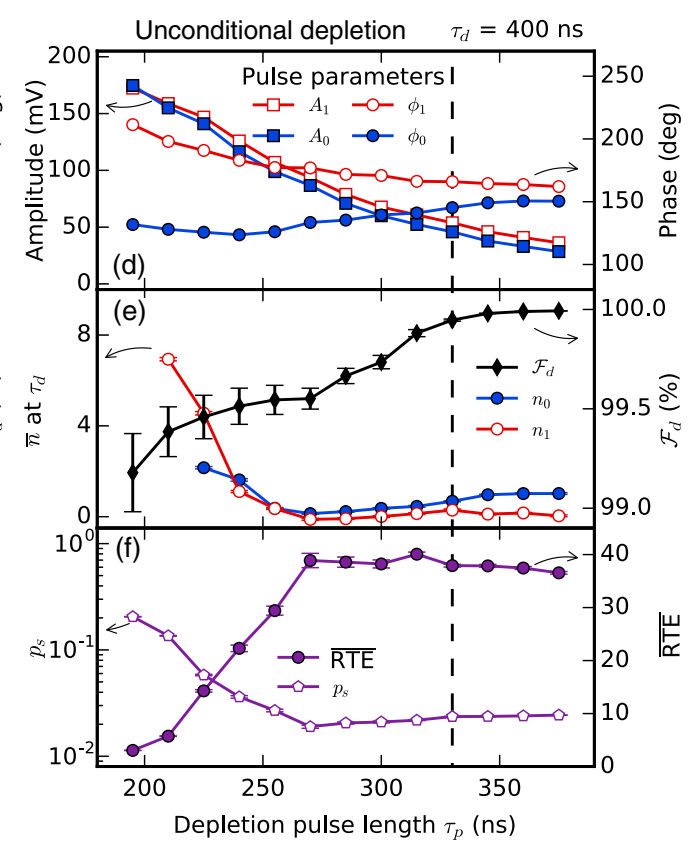

FIG. 4. Characterization of conditional and unconditional depletion as a function of depletion pulse length $\tau_{p}$. The dashed lines indicate the pulse lengths for conditional (unconditional) depletion $\tau_{p}=$ $30 \mathrm{~ns}\left(\tau_{p}=330 \mathrm{~ns}\right)$, used in Figs. 2, 3, and 5. All data are taken at a fixed $\tau_{d}=$ $500 \mathrm{~ns}\left(\tau_{d}=400 \mathrm{~ns}\right)$. (a), (d) Optimal pulse parameters after the two-step optimization protocol. (b),(e) Residual photon number for both qubit states and discrimination fidelity $\mathcal{F}_{d}$ extracted from single-shot readout histograms. (c),(f) Average rounds to an event and per-round probability of a type-s event for emulated QEC as in Fig. 3.

\section{BENCHMARKING DEPLETION METHODS WITH A QEC EMULATION: A NONFLIPPING ANCILLA}

The QEC emulations can be made more sensitive to leftover photons by harnessing the asymmetry of qubit relaxation. Specifically, we change the polarity of the final $\pi / 2$ pulse, ideally returning the qubit to the input state $\Psi_{\text {in }}=|0\rangle$ before measurement and depletion (results for $\Psi_{\text {in }}=|1\rangle$ are discussed in Appendix D). This change removes relaxation as a source of spurious detection events.

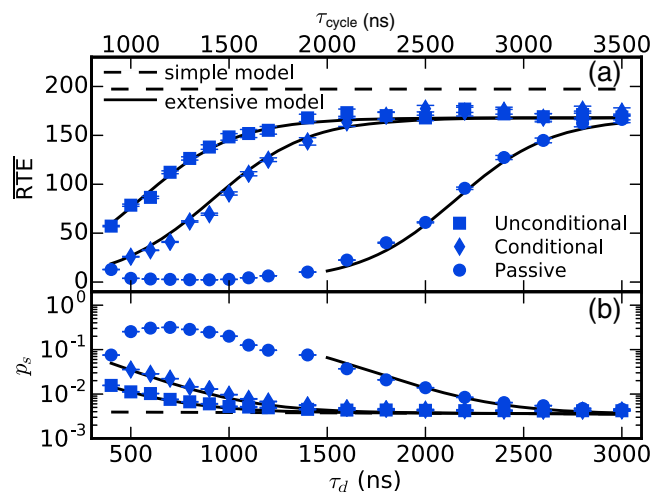

FIG. 5. Emulation of a repeating parity measurement for a nonflipping ancilla starting in $|0\rangle$. This variant uses the sequence of Fig. 3(a) but with opposite polarity on the final $\pi / 2$ pulse in order not to flip the ancilla. (a) The $\overline{\mathrm{RTE}}$ is no longer sensitive to qubit relaxation during $\tau_{d}$ and reaches a ceiling of approximately 168 set by intrinsic decoherence in the coherent step and readout discrimination infidelity. (b) Per-round probability of a type- $s$ event as a function of $\tau_{d}$. Added model curves include the same calibrated errors as in Fig. 3.
For this configuration, unconditional depletion improves the $\overline{\mathrm{RTE}}$ from 1 to 75 at a $1-\mu$ s cycle time (Fig. 5). For longer $\tau_{d}$, the $\overline{\mathrm{RTE}}$ reaches a ceiling of 168 , which is set by intrinsic decoherence in the coherent step and readout discrimination infidelity. Again, the unconditional depletion performs best, but the reduction of the $\overline{\mathrm{RTE}}$ at short $\tau_{d}$ evidences the performance limit reached by our pulses. In a QEC context, the key benefit of active depletion in this nonflipping variant will be an increase in the $\overline{\mathrm{RTE}}$ due to a lower per-cycle probability of data qubit errors, afforded by reducing $\tau_{\text {cycle }}$ by $6 / \kappa$. Evidently, this effect is not captured by our emulation, which is sensitive only to ancilla hardware errors. In quantum-error-correcting schemes, a trade-off needs to be made between shortening cycle times and increasing ancilla fidelity, especially as the different error sources contribute differently to the fidelity of an encoded logical qubit [37].

\section{DISCUSSION AND OUTLOOK}

The RTE experiments motivate two points for discussion and outlook. First, they highlight the importance of digital feedback [38] in QEC to keep ancillas in $|0\rangle$ as much as possible (as used in a cat code [7]). Second, the $\overline{\mathrm{RTE}}$ emerges as an attractive performance metric for every element in the QEC cycle, not just the depletion. The advantage over traditional tune-up methods is the speed gained by not reinitializing in $|0\rangle$ after the measurement [39] and the ability to tune without interrupting ongoing error correction [40].

In summary, we investigate two active methods for fast photon depletion in the nonlinear regime of $\mathrm{CQED}$, relying 
on numerical optimizations to successfully outperform passive depletion by $>6 / \kappa$. Active photon depletion finds applications in quantum-computing scenarios which interleave qubit measurements with coherent qubit operations. Here, we focus on quantum error correction, emulating an ancilla qubit performing repetitive parity checks in a repetition code. Future experiments could map out the theoretically challenging nonlinear readout regime to find the optimum parameters for fast and nondemolition readout and depletion. Motivated by Ref. [36], future experiments will investigate the space of parameters $(\Delta, \kappa, g)$ and especially lower $\Delta$, to pinpoint the optimal conditions for high-fidelity, nondemolition transmon readout in the nonlinear regime. Finally, combining active depletion with Purcell filtering will reduce the QEC cycle time to approximately $500 \mathrm{~ns}$, sufficient to cross the error pseudothreshold in small surface codes at state-of-the-art transmon relaxation times [27].

\section{ACKNOWLEDGMENTS}

We thank S. Visser, J. Somers, L. Riesebos, and E. Garrido Barrabés for contributions to FPGA programming,
K. Lehnert for the parametric amplifier, A. Wallraff for a precision coil, and K. Bertels, E. Charbon, D. Sank, and M. Khezri for discussions. We acknowledge funding from the EU FP7 project ScaleQIT, the Dutch Organization for Fundamental Research on Matter (FOM), an ERC Synergy Grant, the Netherlands Organization for Scientific Research (NWO/OCW), a Marie Curie Career Integration Grant (L. D. C.), and the China Scholarship Council (X. F.).

\section{APPENDIX A: EXPERIMENTAL SETUP}

Figure 6 shows the device and experimental setup, including a full wiring diagram. The chip contains ten transmon qubit-resonator pairs. All experiments presented target pair 2. The experimental setup is similar to that of previous experiments [3] but with an important addition labeled QuTech Control Box. This homebuilt controller, comprised of four interconnected field-programmable gate arrays (Altera Cyclone IV), has digitizing and waveform generation capabilities. The two-channel digitizer samples with eight-bit resolution at $200 \mathrm{MSamples} / \mathrm{s}$. The sixchannel waveform generator produces qubit and resonator pulse envelopes with 14-bit resolution at 200 MSamples/s.

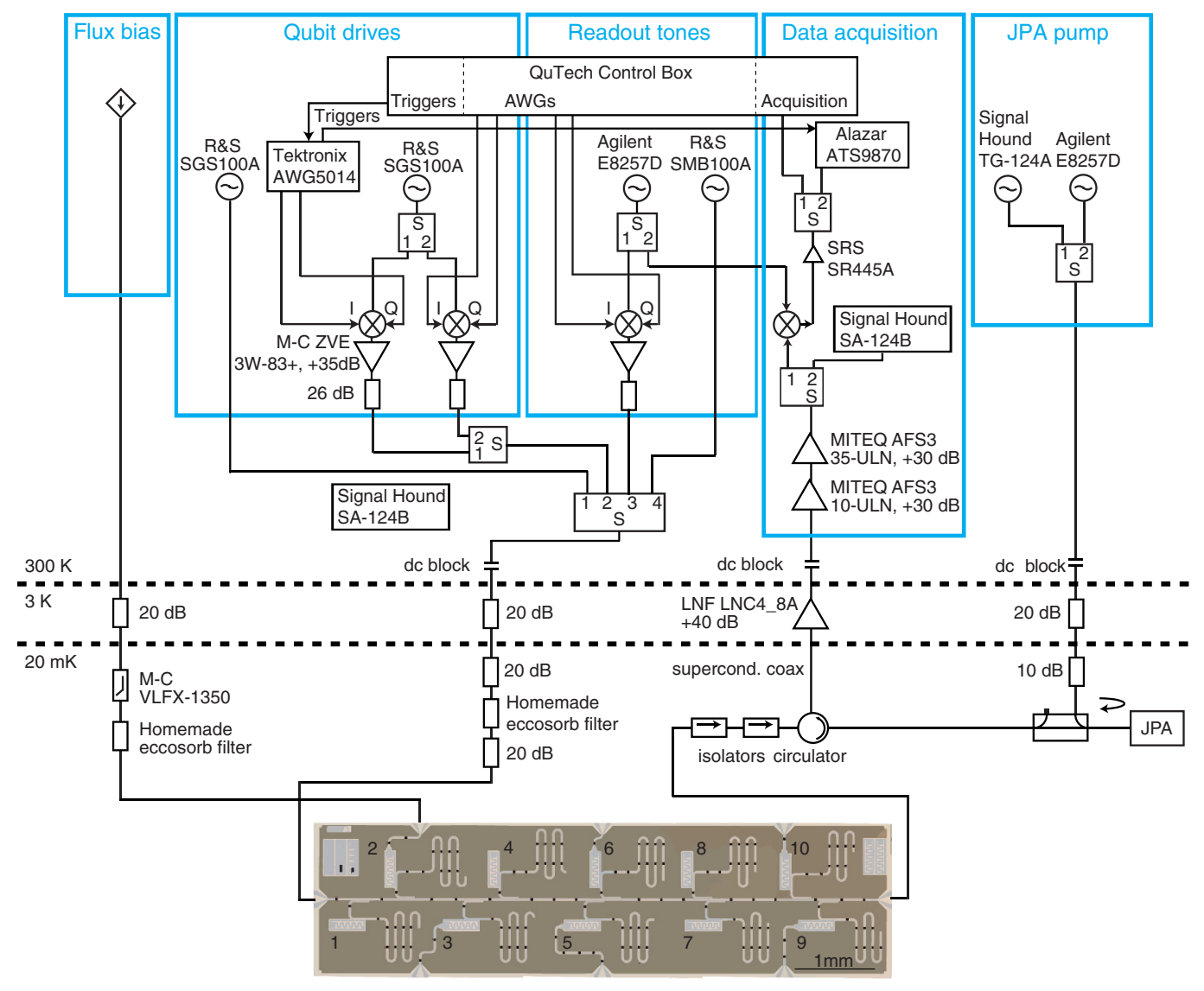

FIG. 6. Photograph of the cQED chip and complete wiring diagram of electronic components inside and outside the ${ }^{3} \mathrm{He} /{ }^{4} \mathrm{He}$ dilution refrigerator (Leiden Cryogenics CF-450). The chip contains ten transmon qubits individually coupled to dedicated readout resonators. All resonators couple capacitively to the common feed line traversing the chip. All data shown correspond to qubit-resonator pair 2 . Dark features traversing the coplanar waveguide transmission lines are $\mathrm{Nb}-\mathrm{Ti}-\mathrm{N}$ bridges which interconnect ground planes and suppress slotline mode propagation. 


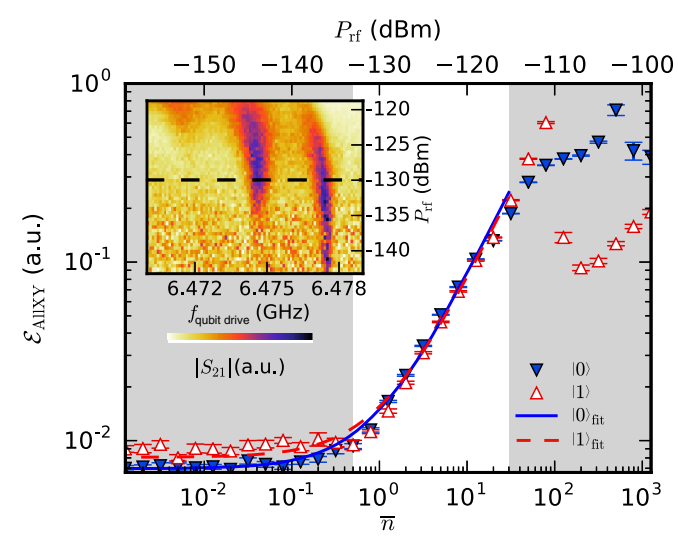

FIG. 7. Calibration of the photon number using the AllXY error. $\mathcal{E}_{\text {AllXY }}$ measured directly after a readout pulse of 1800 -ns duration drives the resonator into a steady-state photon population $\bar{n}$ for input states $|0\rangle$ and $|1\rangle$. The lines show a bilinear fit to the form $\mathcal{E}_{\text {AllXY }}=\alpha \bar{n}+\beta$. Inset: Photon-number-splitting experiment [28] used to calibrate the single-photon power level, $P_{\mathrm{rf}} \sim-130 \mathrm{dBm}$.

\section{APPENDIX B: PHOTON NUMBER CALIBRATION}

Figure 7 contains the calibration of the photon number using the AllXY error $\left(\mathcal{E}_{\text {AllXY }}\right)$ as a detector. $\mathcal{E}_{\text {AllXY }}$ is defined as the average absolute deviation from the ideal two-step result in an AllXY experiment. To calibrate the detector, the resonator is populated using a long (1800-ns) readout pulse with a varying pulse amplitude before measuring the AllXY. This pulse amplitude is converted to an average photon number using the single-photon power that is extracted from a photon-number-splitting experiment. We fit the form $\mathcal{E}_{\text {AllXY }}=\alpha \bar{n}+\beta$ to the data for each input state separately, with $\alpha$ and $\beta$ as free parameters. The best-fit functions are used throughout the experiment to convert $\mathcal{E}_{\text {AlIXY }}$ to $\bar{n}$.

\section{APPENDIX C: NUMERICAL OPTIMIZATION OF DEPLETION PULSES}

This paragraph further describes the optimization of depletion pulses, including the optimization ansatz and convergence criteria. As the optimization algorithm, we use the implementation of Powell's method [24] in SciPy: scipy.optimize.fmin_powell [41].

For conditional depletion, the pulse for $|0\rangle(|1\rangle)$ at frequency $f_{r,|0\rangle}\left(f_{r,|1\rangle}\right)$ is optimized with $\mathcal{E}_{\text {AllXY }}$ as the cost function with the amplitude and phase as free parameters. In the first optimization step with $\tau_{d}=1000 \mathrm{~ns}$, an ansatz pulse is used with a modulation envelope amplitude of $A_{0, \text { init }}=0.035 \mathrm{~V}\left(A_{1, \text { init }}=0.035 \mathrm{~V}\right)$, equal to half the measurement modulation envelope amplitude, and with an initial phase of $\phi_{0, \text { init }}=180^{\circ}\left(\phi_{1 \text {,init }}=180^{\circ}\right)$ with respect to the measurement pulse. After the first iteration, the phase of the pulse is varied with an initial step size of $+10^{\circ}$. After minimizing $\mathcal{E}_{\text {AllXY }}$ by only varying the phase, the algorithm optimizes the amplitude parameter starting with an initial step size of $+10 \mathrm{mV}$. Then, the algorithm chooses nontrivial directions in its parameter space until one of three convergence criteria is met:

(1) The iteration maximum of 300 is reached (reaching this limit indicates a failed convergence).

(2) The change in both parameters is less than 0.001 times the initial step size.

(3) The change in the cost function $\mathcal{E}_{\text {AllXY }}$ is less than 0.00005 .

The second round of optimization at $\tau_{d}=500 \mathrm{~ns}$ uses the final pulse of the first optimization as its starting point and repeats the algorithm with initial step sizes of $1^{\circ}$ and $+1 \mathrm{mV}$. Each iteration takes $12 \mathrm{~s}$, and each optimization step uses approximately 60 iterations to converge. The total two-step procedure takes approximately $48 \mathrm{~min}$ in total for the two pulses combined.

For the unconditional depletion, the sum of $\mathcal{E}_{\text {AllXY }}$ for both input states is used as the cost function. The single four-parameter pulse, composed by summing two square pulses at frequencies $f_{r,|0\rangle}$ and $f_{r,|1\rangle}$, is optimized starting from an ansatz pulse with amplitude and phase parameters $A_{0, \text { init }}=A_{1, \text { init }}=0.035 \mathrm{~V}$ and $\phi_{0, \text { init }}=\phi_{1, \text { init }}=180^{\circ}$. Similar to the two-parameter optimization, the algorithm starts at $\tau_{d}=1000 \mathrm{~ns}$ and starts the first optimization varying one parameter after the other (here, the chosen order is $\left.\phi_{0}, \phi_{1}, A_{0}, A_{1}\right)$. The same initial step sizes and convergence criteria are used as for the conditional depletion, but now a maximum of 500 iterations is chosen. As for the conditional pulses, a second optimization round fine-tunes the pulses, but, because the unconditional pulse is shorter than the sum of latency and the conditional pulse length, a depletion time of $\tau_{d}=400 \mathrm{~ns}$ is used. Each iteration takes 24 s. Each optimization step uses approximately 150 iterations to converge, and the total two-step procedure takes approximately $2 \mathrm{~h}$.

\section{APPENDIX D: CONSTANT EXCITED-STATE QEC EMULATION}

Figure 8 shows the emulated multiround QEC for a nonflipping ancilla when the qubit is initialized in the excited state. This variant of the emulation uses the same sequence as Fig. 5 but with the qubit initialized in $|1\rangle$. Varying $\tau_{d}$, we find the optimum trade-off between errors induced by leftover photons and by relaxation for the three methods. Unconditional depletions performs best, increasing the $\overline{\mathrm{RTE}}$ by a factor of 2.5 with respect to passive depletion. Note that passive depletion produces a spurious increase in the $\overline{\mathrm{RTE}}$ for very short $\tau_{d}$. The high photon number detunes the qubit so much that qubit pulses are inoperative, causing the qubit to remain in the same state and yielding long strings of identical, expected measurement outcomes. 


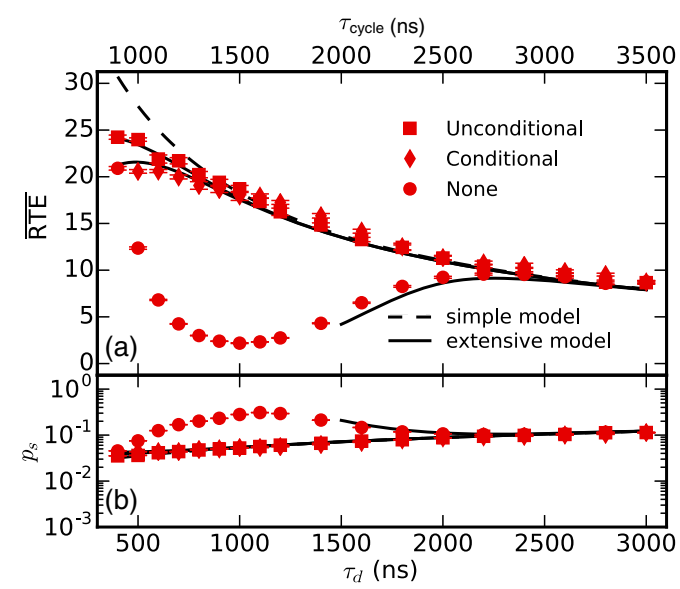

FIG. 8. Emulated multiround QEC for a nonflipping ancilla in $|1\rangle$. This variant of the emulation uses the same sequence as Fig. 5 but with the qubit initialized in $|1\rangle$. (a) Mean rounds to error detection event ( $\overline{\mathrm{RTE}})$ as a function of $\tau_{d}$. (b) Per-round probability of encountering an event of type $s$ as a function of $\tau_{d}$. Added curves correspond to the simple and extensive models described in Appendix E.

\section{APPENDIX E: THEORETICAL MODELS}

We use two models to compare to data in Figs. 3, 6, and 8 labeled simple and extensive. The simple model includes ancilla relaxation and intrinsic dephasing, providing an upper bound for the performance of the emulated multiround QEC circuit. The extensive model further includes an ancilla readout error and detuning and dephasing from the photon-induced ac Stark shift. These models use separately calibrated parameters.

The ancilla sans the photon field is modeled considering amplitude and phase damping as in Ref. [42]. Single-qubit gates are approximated as 40-ns decay windows with perfect instantaneous pulses in the middle. This leads to the following scheme: $\tau_{d}+20 \mathrm{~ns}$ of $T_{1}$ decay, followed by a $\pi / 2$ pulse, then $160 \mathrm{~ns}$ of $T_{2}^{\text {echo }}$ decay (with a $\pi$ pulse in the middle), another $\pi / 2$ pulse, and $20 \mathrm{~ns}$ of $T_{1}$ decay.

The measurement is modeled as a perfect state update $S_{1}$, followed by a $\tau_{r}=300 \mathrm{~ns}$ decay window and a second state update $S_{2}$. The measurement signal is conditioned on both the state post- $S_{1}\left(\mid \psi_{i}\right)$ and post- $S_{2}\left(\mid \psi_{o}\right)$. If $\left|\psi_{i}=\right| \psi_{o}$, no decay occurs, and the incorrect measurement is returned with probability $1-\mathcal{F}_{d}=0.1 \%$ [Fig. 4(b)]. The only other possibility is for a single decay event (as we do not allow excitations). To zeroth order in $\tau_{r} / T_{1} \approx 1 / 800$, this situation has an equal probability of returning either measurement signal.

During the coherent phase, the off-diagonal elements are affected by the photon population. We model this effect following Ref. [35]:

$$
\begin{aligned}
\frac{d \rho^{\mathrm{qb}}}{d t}= & -i \frac{\bar{\omega}_{a}+B}{2}\left[\sigma_{z}, \rho^{\mathrm{qb}}\right]+\gamma_{1} \mathcal{D}\left[\sigma_{-}\right] \rho^{\mathrm{qb}} \\
& +\frac{\gamma_{\phi}+\Gamma_{d}}{2} \mathcal{D}\left[\sigma_{z}\right] \rho^{\mathrm{qb}} .
\end{aligned}
$$

Here, $\mathcal{D}[X]$ is the Lindblad operator $\mathcal{D}[X] \rho=X \rho X^{\dagger}-$ $\frac{1}{2} X^{\dagger} X \rho-\frac{1}{2} \rho X^{\dagger} X, \gamma_{1}=1 / T_{1}$, and $\gamma_{\phi}$ is the pure dephasing rate $\left[\gamma_{\phi}=\left(T_{2}^{\text {echo }}\right)^{-1}-\frac{1}{2} T_{1}^{-1}=(177 \mu \mathrm{s})^{-1}\right] . \bar{\omega}_{a}$ is a constant rotation around the $z$ axis of the Bloch sphere and so is canceled by the $\pi$ pulse in the coherent phase. $\Gamma_{d}=2 \chi \operatorname{Im}\left(\alpha_{0} \alpha_{1}^{*}\right)$ is the measurement-induced dephasing, with $\alpha_{0,1}$ the qubit-state-dependent photon field amplitude and $2 \chi$ the dispersive shift per photon. This contributes a decay to the off-diagonal element of the density matrix during the coherent phase, multiplying it by

$$
\exp \left[-\int \Gamma_{d}(t)\right]
$$

where the integral is taken over the coherent time window. $B=2 \chi \operatorname{Re}\left(\alpha_{0} \alpha_{1}^{*}\right)$ is the ac Stark shift, which detunes the ancilla by an amount equal to the difference in the average photon number over the two parts of the coherent phase. This multiplies the off-diagonal terms by a complex phase

$$
\phi_{\text {Stark }}=\int_{t_{A}} B(t)-\int_{t_{B}} B(t) .
$$

Here, $t_{A}$ and $t_{B}$ are the time windows in the coherent phase on either side of the $\pi$ pulse. The magnitude of the photon fields postdepletion is taken from Fig. 2 and experiences an exponential decay at a rate that is obtained by fitting curves to the same figure. The phase difference between the fields associated with the ground and excited state grows at a rate $2 \chi$, as extracted from Fig. 1. As we do not model photoninduced pulse errors, we restrict our modeling to $\bar{n}<8$, where these effects are negligible.

The experiment is simulated by storing the error-free ancilla population as a unnormalized density matrix and applying repeated cycles of the circuit. At each measurement step, the fraction of the density matrix that corresponds to an event is removed and the corresponding probability stored. The removed fraction of the density matrix is evolved for one more cycle in order to extract the event-type probabilities. This is repeated until the remaining population is less than $10^{-6}$.

[1] M. D. Reed, L. DiCarlo, S. E. Nigg, L. Sun, L. Frunzio, S. M. Girvin, and R. J. Schoelkopf, Realization of three-qubit quantum error correction with superconducting circuits, Nature (London) 482, 382 (2012).

[2] J. Kelly, R. Barends, A. G. Fowler, A. Megrant, E. Jeffrey, T. C. White, D. Sank, J. Y. Mutus, B. Campbell, Y. Chen, Z. Chen, B. Chiaro, A. Dunsworth, I. C. Hoi, C. Neill, P. J. J. O’Malley, C. Quintana, P. Roushan, A. Vainsencher, J. Wenner, A. N. Cleland, and J. M. Martinis, State preservation by repetitive error detection in a superconducting quantum circuit, Nature (London) 519, 66 (2015). 
[3] D. Ristè, S. Poletto, M. Z. Huang, A. Bruno, V. Vesterinen, O.-P. Saira, and L. DiCarlo, Detecting bit-flip errors in a logical qubit using stabilizer measurements, Nat. Commun. 6, 6983 (2015).

[4] J. Cramer, N. Kalb, M. A. Rol, B. Hensen, M. Markham, D. J. Twitchen, R. Hanson, and T. H. Taminiau, Repeated quantum error correction on a continuously encoded qubit by real-time feedback, Nat. Commun. 7, 11526 (2016).

[5] D. Nigg, M. Müller, E. A. Martinez, P. Schindler, M. Hennrich, T. Monz, M. A. Martin-Delgado, and R. Blatt, Quantum computations on a topologically encoded qubit, Science 345, 302 (2014).

[6] A. D. Corcoles, E. Magesan, S. J. Srinivasan, A. W. Cross, M. Steffen, J. M. Gambetta, and J. M. Chow, Demonstration of a quantum error detection code using a square lattice of four superconducting qubits, Nat. Commun. 6, 6979 (2015).

[7] N. Ofek, A. Petrenko, R. Heeres, P. Reinhold, Z. Leghtas, B. Vlastakis, Y. Liu, L. Frunzio, S. M. Girvin, L. Jiang, M. Mirrahimi, M. H. Devoret, and R. J. Schoelkopf, Extending the lifetime of a quantum bit with error correction in superconducting circuits, Nature (London) 536, 441 (2016).

[8] A. Blais, R.-S. Huang, A. Wallraff, S. M. Girvin, and R. J. Schoelkopf, Cavity quantum electrodynamics for superconducting electrical circuits: An architecture for quantum computation, Phys. Rev. A 69, 062320 (2004).

[9] K. D. Petersson, L. W. McFaul, M. D. Schroer, M. Jung, J. M. Taylor, A. A. Houck, and J. R. Petta, Circuit quantum electrodynamics with a spin qubit, Nature (London) 490 , 380 (2012).

[10] T. W. Larsen, K. D. Petersson, F. Kuemmeth, T. S. Jespersen, P. Krogstrup, J. Nygård, and C. M. Marcus, Semiconductor-Nanowire-Based Superconducting Qubit, Phys. Rev. Lett. 115, 127001 (2015).

[11] G. de Lange, B. van Heck, A. Bruno, D. J. van Woerkom, A. Geresdi, S. R. Plissard, E. P. A. M. Bakkers, A. R. Akhmerov, and L. DiCarlo, Realization of Microwave Quantum Circuits Using Hybrid SuperconductingSemiconducting Nanowire Josephson Elements, Phys. Rev. Lett. 115, 127002 (2015).

[12] J. P. Groen, D. Ristè, L. Tornberg, J. Cramer, P. C. de Groot, T. Picot, G. Johansson, and L. DiCarlo, Partial-Measurement Backaction and Nonclassical Weak Values in a Superconducting Circuit, Phys. Rev. Lett. 111, 090506 (2013).

[13] J. E. Johnson, C. Macklin, D. H. Slichter, R. Vijay, E. B. Weingarten, J. Clarke, and I. Siddiqi, Heralded State Preparation in a Superconducting Qubit, Phys. Rev. Lett. 109, 050506 (2012).

[14] D. Ristè, J. G. van Leeuwen, H.-S. Ku, K. W. Lehnert, and L. DiCarlo, Initialization by Measurement of a Superconducting Quantum Bit Circuit, Phys. Rev. Lett. 109, 050507 (2012).

[15] O.-P. Saira, J. P. Groen, J. Cramer, M. Meretska, G. de Lange, and L. DiCarlo, Entanglement Genesis by Ancilla-Based Parity Measurement in 2D Circuit QED, Phys. Rev. Lett. 112, 070502 (2014).

[16] M. D. Reed, B. R. Johnson, A. A. Houck, L. DiCarlo, J. M. Chow, D. I. Schuster, L. Frunzio, and R. J. Schoelkopf, Fast reset and suppressing spontaneous emission of a superconducting qubit, Appl. Phys. Lett. 96, 203110 (2010).
[17] E. Jeffrey, D. Sank, J. Y. Mutus, T. C. White, J. Kelly, R. Barends, Y. Chen, Z. Chen, B. Chiaro, A. Dunsworth, A. Megrant, P. J. J. O’Malley, C. Neill, P. Roushan, A. Vainsencher, J. Wenner, A. N. Cleland, and J. M. Martinis, Fast Accurate State Measurement with Superconducting Qubits, Phys. Rev. Lett. 112, 190504 (2014).

[18] A. A. Houck, J. A. Schreier, B. R. Johnson, J. M. Chow, J. Koch, J. M. Gambetta, D. I. Schuster, L. Frunzio, M. H. Devoret, S. M. Girvin, and R. J. Schoelkopf, Controlling the Spontaneous Emission of a Superconducting Transmon Qubit, Phys. Rev. Lett. 101, 080502 (2008).

[19] J. Gambetta, A. Blais, M. Boissonneault, A. A. Houck, D. I. Schuster, and S. M. Girvin, Quantum trajectory approach to circuit QED: Quantum jumps and the Zeno effect, Phys. Rev. A 77, 012112 (2008).

[20] F. Yan, S. Gustavsson, A. Kamal, J. Birenbaum, A. P. Sears, D. Hover, G. Samach, T. J. Gudmundsen, J. L. Yoder, T. P. Orlando, J. Clarke, A. J. Kerman, and W. D. Oliver, The flux qubit revisited, arXiv:1508.06299.

[21] A. P. Sears, A. Petrenko, G. Catelani, L. Sun, H. Paik, G. Kirchmair, L. Frunzio, L. I. Glazman, S. M. Girvin, and R. J. Schoelkopf, Photon shot noise dephasing in the strongdispersive limit of circuit QED, Phys. Rev. B 86, 180504 (2012).

[22] X. Y. Jin, A. Kamal, A. P. Sears, T. Gudmundsen, D. Hover, J. Miloshi, R. Slattery, F. Yan, J. Yoder, T. P. Orlando, S. Gustavsson, and W.D. Oliver, Thermal and Residual Excited-State Population in a 3D Transmon Qubit, Phys. Rev. Lett. 114, 240501 (2015).

[23] D. T. McClure, H. Paik, L. S. Bishop, M. Steffen, J. M. Chow, and J. M. Gambetta, Rapid Driven Reset of a Qubit Readout Resonator, Phys. Rev. Applied 5, 011001 (2016).

[24] M. J. D. Powell, An efficient method for finding the minimum of a function of several variables without calculating derivatives, Computer Journal (UK) 7, 155 (1964).

[25] J. M. Chow, J. M. Gambetta, E. Magesan, D. W. Abraham, A. W. Cross, B. R. Johnson, N. A. Masluk, C. A. Ryan, J. A. Smolin, S. J. Srinivasan, and M. Steffen, Implementing a strand of a scalable fault-tolerant quantum computing fabric, Nat. Commun. 5, 4015 (2014).

[26] T. E. O'Brien et al. (to be published).

[27] Y. Tomita and K. M. Svore, Low-distance surface codes under realistic quantum noise, Phys. Rev. A 90, 062320 (2014).

[28] D. I. Schuster, A. A. Houck, J. A. Schreier, A. Wallraff, J. M. Gambetta, A. Blais, L. Frunzio, J. Majer, M. H. Devoret, S. M. Givin, and R. J. Schoelkopf, Resolving photon number states in a superconducting circuit, Nature (London) 445, 515 (2007).

[29] C. A. Ryan, B. R. Johnson, J. M. Gambetta, J. M. Chow, M. P. da Silva, O. E. Dial, and T. A. Ohki, Tomography via correlation of noisy measurement records, Phys. Rev. A 91, 022118 (2015).

[30] E. Magesan, J. M. Gambetta, A. D. Córcoles, and J. M. Chow, Machine Learning for Discriminating Quantum Measurement Trajectories and Improving Readout, Phys. Rev. Lett. 114, 200501 (2015).

[31] J. M. Chow, L. DiCarlo, J. M. Gambetta, F. Motzoi, L. Frunzio, S. M. Girvin, and R. J. Schoelkopf, Optimized 
driving of superconducting artificial atoms for improved single-qubit gates, Phys. Rev. A 82, 040305 (2010).

[32] M. Reed, Ph.D. dissertation, Yale University, 2013.

[33] S. Boutin, C. K. Andersen, J. Venkatraman, and A. Blais, in Proceedings of the APS March Meeting 2016 (unpublished).

[34] A. G. Fowler, D. Sank, J. Kelly, R. Barends, and J. M. Martinis, Scalable extraction of error models from the output of error detection circuits, arXiv:1405.1454.

[35] A. Frisk Kockum, L. Tornberg, and G. Johansson, Undoing measurement-induced dephasing in circuit QED, Phys. Rev. A 85, 052318 (2012).

[36] D. Sank, Z. Chen, M. Khezri, J. Kelly, R. Barends, Y. Chen, A. Fowler, E. Jeffrey, E. Lucero, A. Megrant, J. Mutus, M. Neeley, P. Roushan, A. Vainsencher, T. White, B. Campbell, B. Chiaro, A. Dunsworth, C. Neill, P. J. J. O'Malley, C. Quintana, J. Wenner, A. N. Korotkov, and J. M. Martinis, Measurementinduced state transitions in a superconducting qubit: Beyond the rotating wave approximation, arXiv:1606.05721.
[37] B. M. Terhal, Quantum error correction for quantum memories, Rev. Mod. Phys. 87, 307 (2015).

[38] D. Ristè, C. C. Bultink, K. W. Lehnert, and L. DiCarlo, Feedback Control of a Solid-State Qubit Using HighFidelity Projective Measurement, Phys. Rev. Lett. 109, 240502 (2012).

[39] M. A. Rol et al. (to be published).

[40] J. Kelly, R. Barends, A. G. Fowler, A. Megrant, E. Jeffrey, T. C. White, D. Sank, J. Y. Mutus, B. Campbell, Y. Chen, Z. Chen, B. Chiaro, A. Dunsworth, E. Lucero, M. Neeley, C. Neill, P. J. J. O’Malley, C. Quintana, P. Roushan, A. Vainsencher, J. Wenner, and J. M. Martinis, Scalable in-situ qubit calibration during repetitive error detection, arXiv: 1603.03082 .

[41] E. Jones et al., ScIPY: Open source scientific tools for Python (unpublished).

[42] M. A. Nielsen and I. L. Chuang, Quantum Computation and Quantum Information (Cambridge University Press, Cambridge, England, 2000). 